\title{
Regulation of Glycosylation through Glycosyltransferase Shedding by SPPL3
}

\author{
Key Words: Glycosyltransferase, GnT-V, shedding, SPPL3, proteolysis
}

The glycan biosynthesis process involves the transfer of a sugar to an acceptor substrate (glycoprotein or glycolipid) from a donor substrate (sugar nucleotide) by a glycosyltransferase in the ER- or Golgi-lumen. Thus, glycosylation is regulated by the levels or behavior of acceptor substrates, donor substrates and transporters of sugar nucleotides. Among these regulatory factors, glycosyltransferase is undoubtedly one of the most important factors for regulating glycosylation. To date, mechanisms have been well studied that expression and cellular activity of glycosyltransferases are regulated by several factors, including gene expression, subcellular localization or complex formation, and these mechanisms have been shown to underlie glycan changes during differentiation or disease development [Moremen et al. (2012) Nat. Rev. Mol. Cell. Biol. 13, 448-462].

Meanwhile, it is known that glycosyltransferases are cleaved and secreted into culture media and body fluids such as serum. Most glycosyltransferases are type-II membrane proteins with a short N-terminal tail on the cytosolic face and a large catalytic domain on the luminal side, and secreted glycosyltransferases often maintain their catalytic activity with an intact catalytic domain. However, there are essentially no sugar nucleotides in the extracellular space, and little is known about the functions of secreted glycosyltransferases. As an exception, secreted GnT-V (one of GlcNAc transferase) was reported to exhibit an angiogenic potential in a catalytic activity-independent manner [Saito et al. (2002) J. Biol. Chem. 277, 17002-17008]. Furthermore, proteases responsible for glycosyltransferase cleavage and shedding are also poorly understood, with the exceptions of BACE1 for the cleavage of ST6Gall (a sialyltransferase) [Kitazume et al. (2001) Proc. Natl. Acad. Sci. USA 98, 13554-13559] and presenilins for the cleavage of GnT-V [Nakahara et al. (2006) FASEB J 20, 2451-2459]. Very recently, it has been clarified that a GxGD-type protease signal peptide peptidase-like 3 (SPPL3) physiologically cleaves many Golgi-resident glycosyltransferases [Voss et al. (2014) EMBO J 33, 2890-2905; Kuhn et al. (2015) Mol. Cell. Proteomics 14, 1584-1598], leading to a novel concept that shedding of glycosyltransferases is a regulator for glycosylation. The findings of these recent research results will be presented in this review.

SPPL3 is a protease that belongs to the GxGD protease family and was first found with other SPP/SPPLs as a homologous protein to presenilins. These proteases have GxGD and YD motifs that are essential for their proteolytic activity. Presenilins are well known to produce the Alzheimer-related amyloid- $\beta$ peptide and cleave the Notch receptor; however, the physiological substrates and functions of SPPL3 were poorly understood when compared with other family members. Voss et al. first overexpressed SPPL3 in HEK293 cells to examine its functions and found that electromobility of many glycoproteins such as Nicastrin, $N$-cadherin and Lamp2 were shifted downward in SDS-PAGE gel. In contrast, a SPPL3 knockdown caused an upward shift of these glycoproteins. The shifts were removed after cleaving their $N$-glycans by PNGase F, revealing that SPPL3 regulates glycosylation of many glycoproteins. In addition, analysis of SPPL3 knockout (SPPL3-KO) mice gave similar molecular shifts of several glycoproteins in brain, lung, spleen and MEFs (mouse embryonic fibroblasts), indicating that regulation of glycosylation by SPPL3 is a physiological process.

Since SPPL3 was reported to be localized in Golgi apparatus [Friedmann et al. (2006) Nat. Cell. Biol. 8, 843-848], Voss et al. made a reasonable hypothesis that SPPL3 cleaves Golgi-resident glycosyltransferases leading to glycan changes. They particularly focused on an $N$-glycan branching enzyme GnT-V and found that SPPL3 overexpression reduced the amount of full-length intracellular GnT-V and that SPPL3 silencing caused an increase in full-length GnT-V and a decrease in secreted GnT-V. Similar phenomena were also found in SPPL3-KO MEFs, and the changes in secretion were observed for endogenous GnT-V, strongly suggesting that GnT-V is a physiological substrate for SPPL3. Furthermore, the authors expressed recombinant truncated GnT-V with different tags at the $\mathrm{N}$ - and $\mathrm{C}$-termini and found that the cleavage sites were located around the border between the transmembrane and luminal domains.

They also analyzed the change in $N$-glycan pattern in SPPL3overexpressing cells using Nano-LC/MS. Unfortunately, MS/MS data were not shown, and structural identification and quantitativeness were not fully convincing, but the authors claimed that SPPL3 overexpression caused significant changes in $\mathrm{N}$-glycan structures. In particular, reduction of highly branched glycans seemed consistent with the decrease in expression of full-length GnT-V by SPPL3 overexpression. Meanwhile, they also found changes in other glycans, and a GnT-V knockdown caused a less downshift compared with SPPL3 overexpression, suggesting that glycan changes induced by SPPL3 cannot be simply explained by GnT- 
V cleavage. Actually, they biochemically identified three other glycan-related enzymes as SPPL3 substrates (B4GALT1, $\beta 3 \mathrm{GnT} 1$ and $\alpha$-ManI), indicating that SPPL3 has a broad impact on glycan expression.

They also examined the involvement of presenilins because GnT-V was reported to be cleaved by presenilins as described above. However, neither treatment of HEK293 cells with potent presenilin inhibitors nor presenilin deficiency in MEFs showed any change in the secretion of endogenous GnT-V, indicating that SPPL3 but not of presenilins appears to be responsible for GnT$\mathrm{V}$ cleavage at least in these cells. Considering that expression and secretion of GnT-V are involved in cancer development and metastasis, it is possible that other proteases including presenilins are implicated in GnT-V cleavage under pathological conditions or in different cell systems.

In their follow-up paper [Kuhn et al. (2015) Mol. Cell. Proteomics 14, 1584-1598], they tried to comprehensively identify the substrates of SPPL3 using a secretome approach, and here tens of novel substrate (candidates) proteins were identified. They adopted a method called SPECS (secretome protein enrichment with click sugars) that was developed by them as a quantitative proteomic approach for the identification of secreted proteins [Kuhn et al. (2012) EMBO J 31, 3157-3168]. In the SPECS method, cells are treated with a sugar analog azido-sugar (ManNAz), which is incorporated into glycoproteins, and the secreted glycoproteins with azido-sugars are biotinylated by click chemistry, followed by purification and proteomic identification. Although this method has one shortcoming that non-glycosylated secreted proteins are invisible, it does distinguish readily between serum-derived proteins and proteins that are secreted in culture media. By using SPECS, they successfully identified 25 secreted proteins specifically from HEK293 cells overexpressing SPPL3. Interestingly, type-II membrane proteins were highly enriched in identified proteins compared with those in total secreted proteins, strongly suggesting that SPPL3 prefers type-II proteins as a substrate. To be noted, most of the glycosyltransferases are type-II Golgi-proteins, and many glycosyltransferases and related enzymes were enriched in substrate candidates for SPPL3. They also performed the similar SPECS assay using SPPL3-KO MEFs and identified tens of novel glycosylation-related Golgi enzymes as substrates for SPPL3. In addition, analysis of cleaved termini of several secreted proteins suggested that SPPL3 cleaves a putative motif around the border between the transmembrane and luminal domains.

In summary, these results strongly suggest that SPPL3 serves as a physiological sheddase for many glycosyltransferases. Although SPPL3-KO mice show infertility and growth defect phenotypes [Tang et al. (2010) Nat. Biotechnol. 28, 749-755], the reason for these defects have not been clarified yet. Now, many physiological substrates for SPPL3 are known, and its physiological functions will probably be unveiled from a glycobiology point of view. Future studies on SPPL3 are expected to elucidate why so many glycosyltransferses are cleaved and secreted out.

\section{Reported by Yasuhiko Kizuka}

Disease Glycomics Team, RIKEN,

2-1 Hirosawa, Wako-shi, Saitama 351-0198, Japan

E-mail: y.kizuka@riken.jp

FAX: +81-48-467-9617 